\title{
Recurrence Following Anastomotic Leakage After Surgery for Carcinoma of the Distal Esophagus and Gastroesophageal Junction: A Systematic Review
}

\author{
PAOLO AURELLO, GIAMMAURO BERARDI, GIOVANNI MOSCHETTA, \\ MATTEO CINQUEPALMI, LAURA ANTOLINO, GIUSEPPE NIGRI, \\ FRANCESCO D'ANGELO, STEFANO VALABREGA and GIOVANNI RAMACCIATO
}

General Surgery Unit, Department of Medical and Surgical Sciences and Translational Medicine, St. Andrea University Hospital, Sapienza University of Rome, Rome, Italy

\begin{abstract}
Background: Esophageal cancer is the ninth most common cancer. The only potentially curative treatment is surgical resection, which unfortunately is still associated with major complications, the most important being anastomotic leakage, currently with an overall rate of up to $26 \%$ morbidity. The aim of this systematic review was to evaluate the relationship between anastomotic leakage and recurrence of disease. Materials and Methods: A literature search was systematically performed. Seven out of 312 articles dated between 2009 and 2018 fulfilled the selection for a total of 5,433 patients. Results: The frequency of anastomotic leakage ranged from 7.2 to 11.2\%. Patients affected by anastomotic leakage had a recurrence rate of 9-56\%. Conclusion: Closer follow-up or even more aggressive oncological therapy should be considered for patients affected by anastomotic leakage after surgery for carcinoma of the distal esophagus and gastroesophageal junction.
\end{abstract}

Esophageal cancer is reportedly the ninth most common cancer and the sixth most common cause of cancer-related death globally, with a 5-year survival rate of about $20 \%$, therefore still representing an important oncological issue (1).

This article is freely accessible online.

Correspondence to: Dr. Cinquepalmi Matteo, Via di Grottarossa 1037, 00189, Rome, Italy. Tel: +39 0633776746, Fax: +39 0633775649, e-mail: matteo.cinquepalmi@uniroma.it

Key Words: Gastroesophageal junction carcinoma, esophageal carcinoma, anastomotic leakage, recurrence, disease-free survival, review.
Esophageal cancer is classified as squamous cell carcinoma or adenocarcinoma. Both occur more commonly in men. Squamous cell carcinoma is the most common histology in Asia, while adenocarcinoma is most common in North America and Western European countries, according to National Comprehensive Cancer Network Guidelines Version 1 Esophageal and Esophagofastric Junction Cancers, 2017 (2).

In the revised American Joint Committee on Cancer (AJCC) staging system, tumors whose midpoint is in the lower thoracic esophagus, gastro-esophageal junction (GEJ) or within the proximal $5 \mathrm{~cm}$ of the stomach extending into the GEJ or esophagus (Siewert types I and II) are classified as adenocarcinomas of the esophagus for staging purposes. All other cancer located more than $5 \mathrm{~cm}$ distal to the GEJ, or within $5 \mathrm{~cm}$ of the GEJ but not extending into the GEJ or esophagus (Siewert type III) are staged using the gastric cancer staging system (3).

The only potentially curative treatment is surgical resection, which unfortunately is still associated with major complications; for example, the frequency of anastomotic leakage (AL), one of the most important causes of morbidity, in the literature varies between 7.2 and $13.6 \%$ (4).

$\mathrm{AL}$ affects long-term survival in patients after colorectal cancer surgery because of increased hospital stay, higher reoperation rates, wound infection, pneumonia and sepsis. This can eventually result in failure or delay in referring patients for adjuvant chemotherapy (5). The effect of morbidity after esophageal and GEJ surgery on oncological outcomes is not well established, with fragmentary and discordant results (6).

Due to the lack of consensus, the aim of this systematic review was to evaluate the relationship between AL and recurrence of disease, by pooling the results currently available in the literature. 
Table I. Characteristics of the included studies (all retrospective clinical studies).

\begin{tabular}{|c|c|c|c|c|c|c|c|c|}
\hline Authors, year (Ref) & Country & Histology & $\begin{array}{l}\text { Study } \\
\text { period }\end{array}$ & $\begin{array}{c}\text { Patients, } \\
n\end{array}$ & $\begin{array}{c}\mathrm{AL} \\
\mathrm{n}(\%)\end{array}$ & $\begin{array}{c}\text { Median age, } \\
\text { years }\end{array}$ & $\begin{array}{c}\text { Gender, } \\
\mathrm{M} / \mathrm{F}\end{array}$ & $\begin{array}{l}\text { NOS } \\
\text { score }\end{array}$ \\
\hline Lerut et al., 2009 (21) & Belgium & $\mathrm{S}, \mathrm{AC}$ & 2005-2006 & 138 & $10(7.2)$ & 63 & $111 / 27$ & 8 \\
\hline Hii et al., 2013 (22) & Australia & $\mathrm{S}, \mathrm{AC}$ & $1998-2011$ & 618 & $69(11.2)$ & NR & $523 / 95$ & 7 \\
\hline Markar et al., 2015 (23) & France & $\mathrm{S}, \mathrm{AC}$ & $2000-2010$ & 2439 & $208(8.5)$ & 60.6 & $2000 / 439$ & 9 \\
\hline Kofoed et al., 2015 (24) & Denmark & $\mathrm{S}, \mathrm{AC}$ & 2003-2011 & 1085 & $93(8.5)$ & 63 & $860 / 225$ & 8 \\
\hline Andreou et al., 2016 (25) & Germany & $\mathrm{S}, \mathrm{AC}$ & 2005-2012 & 471 & $41(8.7)$ & 65 & $352 / 119$ & 8 \\
\hline Tam et al., 2016 (26) & US & $\mathrm{AC}$ & $1997-2010$ & 334 after PMS & 95 (NR) & 68 & $644 / 119$ & 7 \\
\hline Belmouhand et al., 2017 (27) & Denmark & $\mathrm{AC}, \mathrm{GEJ}$ & 2003-2011 & 348 & $36(10.3)$ & 68 & $297 / 51$ & 9 \\
\hline
\end{tabular}

AL: Anastomotic leakage; NL: no leakage; AC: adenocarcinoma; S: squamous cell carcinoma; GEJ: gastroesophageal junctional; NR: not reported; NOS: Newcastle-Ottawa scale; PMS: propensity match score.

\section{Materials and Methods}

Search strategy. We conducted a systematic search of PubMed, EMbase, Cochrane Library, CILEA Archive, BMJ Clinical Evidence and Up ToDate databases with the following search terms: "Cancer" AND "esophageal" (or "esophagus") AND "esophagogastric junction" AND "anastomotic leakage" (or fistula or dehiscence) AND "recurrence" or "relapse" or "disease-free survival"). All reference lists from the studies selected by electronic searching were cross-checked for further identification of other relevant studies. Unpublished reports were excluded.

Study selection. The search was limited to articles written in English from January 1998 through April 2018. The complete article was retrieved when the information in the title or abstract appeared to meet the objective of this review. In addition, the reference lists of the studies thus obtained were searched manually for any relevant articles not found in the computerized search. We considered prospective and retrospective studies, whether multicentered or not, on esophagectomy performed for esophageal and GEJ cancer. The following inclusion criteria were set: i) English language study; ii) open or minimally invasive surgery with curative intent (with histological confirmation of R0 status; iii) cases of GEJ adenocarcinoma or esophageal adenocarcinoma or esophageal squamous cell carcinoma; iv) studies mentioning recurrence rates with or without disease-free survival (DFS). The following exclusion criteria were set: i) Treatment for disease recurrence; ii) case report; iii) letter; iv) review.

Data extraction and quality assessment. Two Authors (GB, GM) independently screened the articles by title, abstract and key words, and then selected and analyzed the articles. Any disagreement was resolved by discussion or with the opinion of the senior Author (PA).

PRISMA statement guidelines for conducting and reporting systematic reviews were followed (7). The research protocol was registered at the International Prospective Register of Systematic Reviews (http://www.crd.york.ac.uk/PROSPERO) with the following registration number: CRD42018104854 (8). The Newcastle-Ottawa Scale was the instrument used for quality assessment; this was developed for nonrandomized studies in order minimize bias risk in systematic review and is recommended by the Cochrane Collaboration (9). Three broad perspectives are used to judge a study evaluating selection of the study group, comparability, ascertainment of either the exposure or outcome of interest for casecontrol or cohort studies, respectively (10). Newcastle-Ottawa Scale quality assessment of the included studies is presented in Table I.

\section{Results}

Study selection. The literature search yielded 10,824 articles; 10,512 were not considered suitable for the abstract or integral reading, including duplicate removal; 312 titles and abstracts were then reviewed (Figure 1). Of these, 295 were excluded because they did not conform to our review requirements. Finally, only 17 articles were selected for fulltext review and, of these, 10 more were excluded: One because it reported too few cases to draw conclusions, as the authors themselves declared (11); two more because they did not report outcomes of interest, such as overall survival or disease-free survival $(12,13)$; five because they did not report recurrence rates in patients with occurrence of $\mathrm{AL}$, although hypothesizing a possible relationship (14-18); and two because they did not report recurrence rates at all (19-20). There was no disagreement regarding eligibility of full-text articles (Cohen kappa=1). Finally, a total of seven articles fulfilled the selection criteria and were therefore included in this systematic review (21-27); this pool of articles were retrospective reports. Characteristics of the included studies are presented in Table I, which shows patient information, and in Table II, which demonstrates outcomes of interest.

All these articles included a total of 5,433 adult patients who underwent surgical treatment for esophageal and GEJ cancer. The studies included were assessed for quality and the results are presented in Table I. Outcomes of interest of each single study are summarized in Tables II and III.

Age at presentation. All the articles in this systematic review reported the age of patients at presentation of the disease, except that by Hii et al. (22). The median age ranged from 60.6 to 68 years and was not correlated with AL incidence (Table I). 


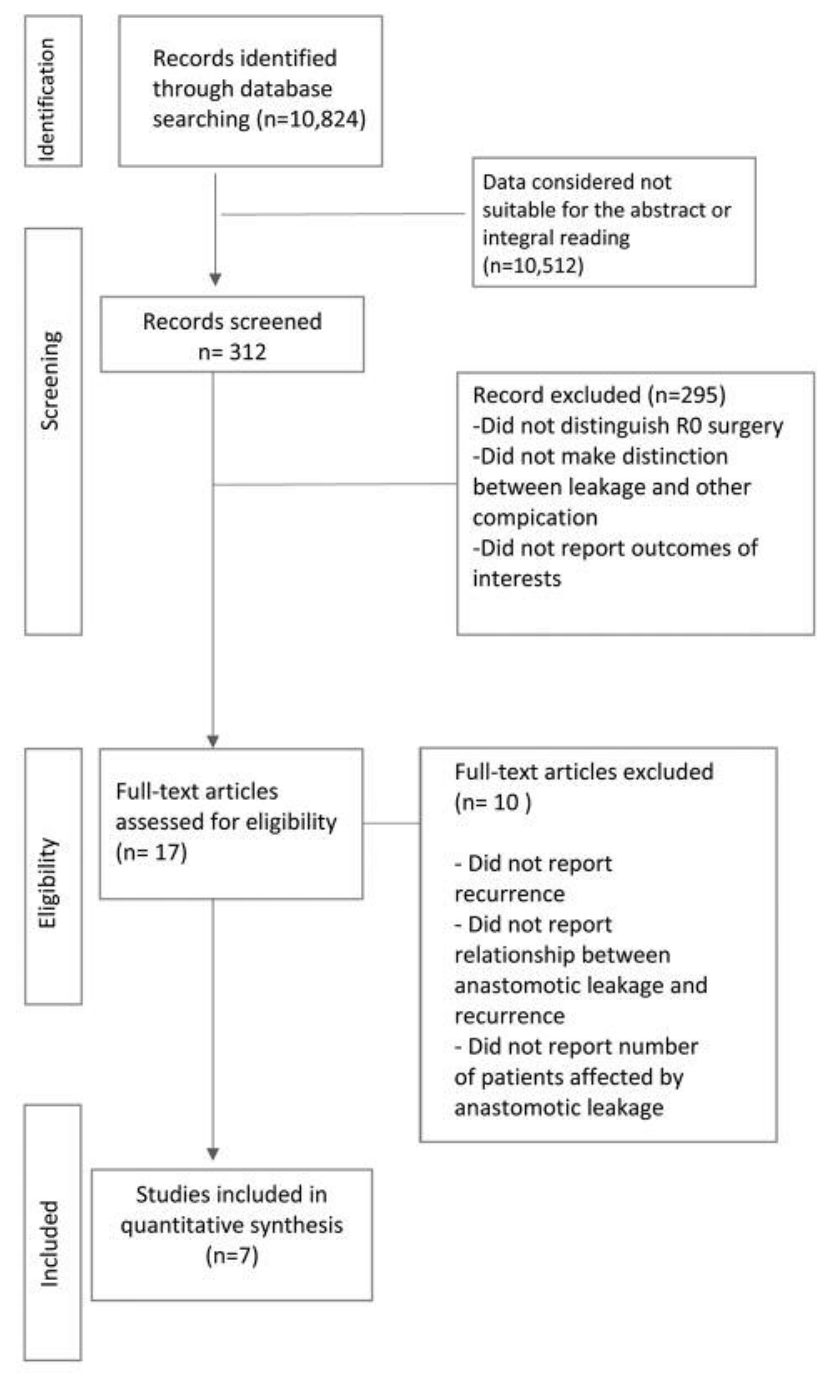

Figure 1. Study selection algorithm.

Recurrence and $A L$. Among the 5,433 cases, the total number of recurrences, cases of AL and the associations between these two factors are highlighted in Tables II and III, article by article. Table III present a summary of the data of interest. Three out of seven studies dealt only with patients with recurrence $(22,23,26)$; the others included cohorts of patients with or without recurrence or AL (21, $24,25,27)$.

AL rates patients with recurrence was 9-13\%. All the included studies reported oncological outcomes of the study cohorts, however, we focused our work on the association between the following two variables: Recurrence and disease-free survival, as highlighted in Table III.

\section{Discussion}

Some research studies have shown a relationship between surgery and oncological prognosis and its possible role in growth and metastasis of malignant tumor cells (27), although this relationship is still not clear. In our review, we found patients presenting with recurrence after AL following surgery performed for esophageal or GEJ tumor. The definition of this relationship is crucial in order to select the population of patients susceptible to recurrence who might benefit from more intensive follow-up. Despite the few articles on the relation between $\mathrm{AL}$ and recurrence, this relationship can be speculated. In our study, we found, as demonstrated by others, many factors could be implied in this relationship, including cell-mediated immunity, cytokines, and inflammatory pathway (27), thus allowing us to have a biological basis to the relationship sought in the articles, one by one.

Regarding the possible pathophysiological basis of the correlation between AL and recurrence, in a literature review, Beecher et al. already demonstrated how surgical trauma, inflammatory response, intraoperative tumor manipulation, growth factors released during the procedure and tumor growth have significant relevance in recurrence (28).

Surgery itself can lead to suppression of cell-mediated immunity, and diffusion of malignant cells into the bloodstream, therefore increasing the metastatic potential, and non-locoregional recurrence was also taken into consideration $(23,27)$.

Among studies taken into account, there is no uniqueness of results regarding the role of $\mathrm{AL}$ in influencing recurrence or not. However, all the most statistically significant studies taken into consideration by our group reported the evidence of an impact of AL on recurrence. Lerut et al. in their work including 138 patients did not demonstrate a statistically significative link between AL and recurrence ( $p=0.7077$ ), but this result might, partly, be due to few patients taken into account (21). In contrast, their study reported a significant correlation between occurrence of complications and the timing of recurrent disease, with due regard more to the severity of the complication, rather than AL itself. Hii et al. described the impact of postoperative morbidity on disease-free and overall survival after esophagectomy for 618 patients. They reported both medical and surgical complications, sorting these into independent categories, such as AL, in order to evaluate the impact on median survival. However, with the occurrence of complications, their study shown only longer duration of inpatient stay, without a clear influence on disease-specific survival. Therefore, they reported two main groups of patients, those who received neoadjuvant therapy followed by surgery and those who underwent surgery alone. The interesting aspect of this study was how for the surgery-alone group, the univariate analysis showed a significant association, but not the 
Table II. Primary outcomes of each included study.

\begin{tabular}{|c|c|c|c|c|c|c|}
\hline \multirow{2}{*}{ Authors, year (Ref) } & \multirow{2}{*}{$\begin{array}{l}\text { Follow-up duration, } \\
\text { months }\end{array}$} & \multirow[b]{2}{*}{$\mathrm{AL}, \mathrm{n}$} & \multicolumn{2}{|c|}{ 5-Year DFS (\%) } & \multicolumn{2}{|c|}{ OS, months } \\
\hline & & & AL & NL & AL & NL \\
\hline Lerut et al., 2009 (21) & $(32-72)$ & 10 & NR & NR & NR & NR \\
\hline \multicolumn{7}{|l|}{ Hii et al., 2013 (2) } \\
\hline Surgery alone & $51(2-151)$ & 69 & $22 \%(p=0.251 \mathrm{~m})$ & $65 \%$ & $22(p=0.516 \mathrm{~m})$ & 52 \\
\hline Neoadjuvant & & & $32 \%(p=0.674 \mathrm{u})$ & $36 \%$ & $41(p=0.818 u)$ & 41 \\
\hline Markar et al., 2015 (23) & 54 & 208 & $34.9 \%(p=0.005)^{*}$ & $47.9 \%$ & $35.8(p=0.002)^{*}$ & 54.8 \\
\hline Kofoed et al., 2015 (24) & 29 & 93 & 27 & $39 \%$ & NR & NR \\
\hline Andreou et al., 2016 (25) & $35(1-101)$ & 41 & $35 \%(p=0.037)^{*}$ & $58.8 \%$ & $39 \%(p=0.001)^{*}$ & $61 \%$ \\
\hline Tam et al., 2016 (26) & 35.6 & 95 & NR & NR & $\begin{array}{c}26.8(9.2-99) \\
(p=0.014)^{*}\end{array}$ & $\begin{array}{c}43.1 \quad(\mathrm{IQR}=18-178) \\
(p=0.014)\end{array}$ \\
\hline Belmouhand et al., 2017 (26) & $38.5(18-69)$ & 36 & NR & NR & NR & NR \\
\hline
\end{tabular}

AL: Anastomotic leakage; DFS: disease-free survival; IQR: interquartile range; NL: no leakage; NR: not reported; OS: overall survival; mmultivariate analysis; uunivariate analysis. Data are median values (range). ${ }^{*} p$-Value reported in the reference article.

Table III. Relationship between recurrence and anastomotic leakage.

\begin{tabular}{|c|c|c|c|c|}
\hline Authors, year (Ref) & No. of patients & Recurrence, n (\%) & No recurrence, $\mathrm{n}(\%)$ & $p$-Value \\
\hline Lerut et al., 2009 (21) & 138 & $\begin{array}{c}\text { Total: } 47 \\
\text { AL: } 4(9 \%)\end{array}$ & $\begin{array}{l}\text { Total: } 89 \\
\text { AL: } 6(7 \%)\end{array}$ & 0.7077 \\
\hline Hii et al., 2013 (22) & 618 & DFS: AL: $32 *$ & NL: 36 & 0.674 \\
\hline Markar et al., 2015 (23) & $\begin{array}{r}208 \\
2331\end{array}$ & $\begin{array}{l}\text { AL (5 years): } 56.1 \% \\
\text { NL (5 years): } 48.5 \%\end{array}$ & NR & 0.009 \\
\hline Kofoed et al., 2015 (24) & 1085 & $\begin{array}{c}\text { Total: } 369 \\
\text { AL: } 39(11 \%) \\
\text { NL: } 330(89 \%)\end{array}$ & $\begin{array}{c}\text { Total: } 716 \\
\text { AL: } 54(8 \%) \\
\text { NL: } 662(92 \%)\end{array}$ & 0.004 \\
\hline Andreou et al., 2016 (25) & 471 & $\begin{array}{c}\text { Total: } 144 \\
\text { AL: } 19(13 \%)\end{array}$ & $\begin{array}{c}\text { Total: } 327 \\
\text { AL: } 23(7 \%)\end{array}$ & 0.048 \\
\hline Tam et al., 2016 (26) & 334 & $\begin{array}{l}\text { AL: } 38 \\
\text { NL: } 30\end{array}$ & NR & 0.228 \\
\hline Belmouhand et al., 2017 (27) & 348 & $\begin{array}{c}\text { Total: } 120 \\
\text { AL: } 15(12.5 \%) \\
\text { NL: } 107(87.5 \%)\end{array}$ & $\begin{array}{c}\text { Total: } 228 \\
\text { AL: } 21(9.2 \%) \\
\text { NL: } 207(90.8 \%)\end{array}$ & $\begin{array}{c}0.34 \\
\text { (univariate } \\
\text { analysis) }\end{array}$ \\
\hline
\end{tabular}

AL: Anastomotic leakage; DFS: disease-free survival; NL: non anastomotic leakage; NR: non reported. *According to AL presence, recurrence not specified.

multivariate one. In the group treated with neoadjuvant therapy followed by surgery, both univariate and multivariate analyses were negative (22). Markar et al. intended to demonstrate exactly the impact of severe esophageal anastomotic leak upon locoregional cancer recurrence (23). They considered severe leak as a symptomatic leakage of intrathoracic anastomosis, defined as Clavien-Dindo III/IV (28). In the final analysis, 2,439 patients were included, among them 1,129 patients underwent neoadjuvant treatment. The total number of ALs was 208 , with a recurrence rate of $56.1 \%$. On the other hand, the recurrence rate among 2,231 patients who did not experience AL was significantly lower at about $48.5 \%$ $(p=0.009)$. Indeed, the subsequent statistical study showed greater overall recurrence [odds ratio $(\mathrm{OR})=1.35,95 \%$ confidence intervaI $(\mathrm{CI})=1.15-1.73 ; p=0.011]$, including both locoregional $(\mathrm{OR}=1.56 ; 95 \% \mathrm{CI}=1.05-2.24 ; p=0.030)$ and mixed $(\mathrm{OR}=1.81,95 \% \mathrm{CI}=1.20-2.71 ; p=0.014)$, in patients affected by $\mathrm{AL}$, therefore agreeing with our hypothesis. In the same way as us, they sought pathophysiological reasons to explain this relationship, stressing the importance of the proinflammatory environment, with many chemical mediators, such as cytokines and acute-phase reactants, which may be 
responsible for tumor cell proliferation and progression to metastasis. In conclusion, Markar et al. showed statistically significant evidence on how AL adversely affects cancer prognosis, thereby increasing recurrence, providing therefore strong evidence in terms of the number of patients involved in their large multicenter study (23). In their study, 33 patients $(23.9 \%)$ underwent induction chemotherapy or radiotherapy. They did not specify the number of patients with AL affected by recurrence or vice versa. Therefore, it was not possible to speculate on its influence on recurrence rates. Using Cox proportional-hazards regression and evaluating timing of the recurrences, compared with patients with grade zero ClavienDindo score, a statistically significantly higher recurrence rate was recorded in patients with higher Clavien-Dindo grades. They also proposed Clavien-Dindo classification as a interesting tool that can enable precise documentation of postoperative complications, based on severity.

Kofoed et al. included 1,085 patients in their study. They reported the impact of intrathoracic AL after curative resection of esophageal or GEJ cancer, investigating whether $\mathrm{AL}$ was associated with increased risk of recurrence. They demonstrated a significantly increased risk of cancer recurrence after intrathoracic AL [hazard ratio $(\mathrm{HR})=1.63$; $95 \% \mathrm{CI}=1.17-2.29, p=0.004]$ in patients who underwent intended curative resection for gastroesophageal cancer, supporting our hypothesis. They explained this relationship by attributing a modified immunological response as being responsible for providing better conditions for growth of circulating tumor cells (23). In their study, AL was associated with a significantly higher risk of recurrence $(\mathrm{HR}=1.63,95 \% \mathrm{CI}=1.17-2.29 ; p=0.004)$ and all-cause mortality $(\mathrm{HR}=1.57,95 \% \mathrm{CI}=1.23-2.05, p<0.0001)$. They attributed this poor outcome to inflammatory response, generated by AL. Therefore, mediastinitis, infection in the pleural cavity, and septicaemia might also facilitate the metastatic cascade that eventually leads to disease recurrence. They furthermore highlighted how the possible delaying of postoperative oncology therapy could be a critical factor implied in an increased risk of recurrence.

Another included retrospective study, by Andreou et al., was aimed at investigating whether AL could predict diminished long-term survival after resection for gastric and esophageal cancer, also reporting the correlation between leakage and recurrence, taken into account in our study. This study also included gastric cancer, which could be a potentially source of bias for our research. They included 471 patients, $47 \%$ of them affected by esophageal tumor; $12.9 \%$ suffered AL. In their statistical analysis of patients with esophageal cancer, they confirmed the role of AL as a risk factor of increased probability of recurrence $(\mathrm{HR}=1.74$, 95\% CI=1.03-2.96, $p=0.037$ ), therefore confirming our suggestion of an association between $\mathrm{AL}$ and recurrence: in their multivariate analysis for predictors of disease-free survival, $\mathrm{AL}(p=0.037)$ was associated with a high risk of recurrence $(25)$.

Like us, they inquired about biological features, raising the possibility that implied generalized immune suppression, or the depression of cell-mediated immunity (especially natural killer cells and cytotoxic T-lymphocytes) might be invoIved in promoting spread and growth of micrometastases. They also attributed the development of a proinflammatory environment, which can promote cancer cell survival and proliferation, to AL. All these factors were also considered to be involved in increased risk of systemic recurrence. Indeed, they considered the risk of systemic recurrence as the most relevant, the liver being the most common site, therefore suggesting the possible systemic effects of circulating factors. As well another article cited (26), they also considered delayed administration of adjuvant systemic therapy responsible for increased risk of recurrence.

An interesting work written by Tam et al. included 334 patients and was carried out in the USA (26). All patients included in their study underwent minimally invasive surgery. The study was particularly focused on the rate of infection and the correlation this had with recurrence, taking advantage of propensity score matching, a tool used to create similar groups in order to reduce errors. We included this work for the important considerations it makes regarding the correlation between AL and outcome: There was no statistically significant difference in recurrence rates between propensity-matched cohorts with and those without AL $(\mathrm{n}=38$ versus $\mathrm{n}=30 ; p=0.228)$, nor in disease-free survival (Breslow $p=0.158$ ), or median time to recurrence in those whose disease recurred (14.14 versus 15.2 months; Wilcoxon signed-rank test $p=0.552)$. In the propensity-matched AL data set, the median time to follow-up was 35.6 months; $75 \%$ of matched patients with AL died during follow-up $(n=71)$ compared with $64 \%(n=61)$ of patients without. Median overall survival was 26.8 months (interquartile range $=9.2-99$ months) versus 43.1 months (interquartile range $=18-178$ months) in the group without AL (Breslow $p=0.014)$. After adjusting for recurrence, $\mathrm{R} 0$ resection status, age at operation, positive circumferential margin, age-adjusted Charlson comorbidity index score, sex, body mass index, induction therapy, and pathological stage (American Joint Committee on Cancer, seventh edition), postoperative AL was independently associated with a $60 \%$ increased hazard of death during follow-up compared with patients without $\mathrm{AL}(\mathrm{HR}=1.60,95 \% \mathrm{CI}=1.12-2.89)(26)$.

The last study, performed by Belmouhand et al., included 348 patients and evaluated recurrence following curativelyintended surgery for GEJ adenocarcinoma. Oncological perioperative chemotherapy was administered to 220 patients. This study was carried out in Denmark, like another included work by Kofoed et al. (24). However, they were located at different centers. Furthermore, Belmouhand 
et al. included only junctional adenocarcinoma, considering a smaller number of patients (348 vs. 1,085). The information of our interest showed how recurrence occurred in 120 patients $(34.5 \%)$, with 32 local $(9.2 \%)$, and 88 distant (25.3\%) recurrences, verified with biopsy. Nevertheless, they did not find any factors associated with site-specific recurrence. Only a trend toward local recurrence was identified for patients who had $\mathrm{AL}$ $(\mathrm{HR}=2.64,95 \% \mathrm{CI}=0.89-7.86 ; p=0.08)(27)$. It is worthy of mention how the other Danish study (24), including a larger cohort of patients, showed not a trend but instead a clear link between AL and local recurrence, as mentioned in our discussion above.

Furthermore, we did not find correlation in the selected studies on the relationship between T-stage and adjuvant therapy, nor between stage and adjuvant therapy. It is interesting to note that four studies found no relationship between AL and recurrence rate; this could be due to the number of patients included. It is important to underline that in the work by Tam et al. (26), once the corrective factors for bias were applied, AL was proven to reduce long-term survival, even not correlated with the recurrence rate. Moreover, all the most numerically important studies show how the data support our hypothesis. The total number of patients who experienced AL was 5,433 among the included studies: 3,995 experienced recurrence versus 1,438 who did not. The pathophysiological basis which supports our hypothesis is that esophageal surgery lead to immunosuppression, and a cytokine storm contributes to creating a microenvironment that favors a more rapid recurrence than the group not exposed to this particular complication. Different systems of AL evaluation were used in the included studies. Lerut et al. used a modified version of the Clavien classification, with different well-defined grades of severity (21). In the study by Markar et al., only clinically significant severe esophageal AL was considered (23). Severe esophageal AL was defined as a symptomatic (mediastinal abscess, mediastinitis or digestive content in the chest drain) disruption of the intrathoracic anastomosis, classified as grade III or IV according to the Clavien-Dindo classification (29). For Kofoed et al., AL was defined radiologically, or using an acute computed tomography scan performed because of clinical signs of leakage (fever, chest pain, saliva or gastrointestinal content through chest drain) (24). Hii et al. also used the same methods of AL diagnosis (22). For Andreou et al. AL was identified by routine radiocontrast agent swallow examination, also including nonclinical AL (25). Tam et al., on the other hand, considered grade II-IV AL or conduit leak (26). Belmouhand et al. evaluated AL with either an oral X-ray with contrast agent, computed tomography with an oral contrast agent, or gastroscopy. Patients with AL were categorized as requiring an intervention (Clavien-Dindo score $\geq 3$ ) (27).
Considering how AL might influence prognosis, we believe the technique used for anastomosis is worthy of mention. Therefore, we comment on an interesting article written by Wong et al. (30) This study focused on how, after discouraging results after the introduction of the circular stapler, a single layer of continuous suture was reconsidered over time as the best technique for achieving the best results. Using this technique, the clinical leakage rate fell to $2 \%$, thus demonstrating how this hand-sewn technique was superior. Wong et al. emphasized the technical aspect, specifying in particular that the two parts in this hand-sewn technique should be lightly apposed, and not approximated tightly, in order to avoid edge strangulation. They also demonstrated how visual satisfaction could predict outcome by defining anastomosis as "'Simple' when it was straightforward and looked pleasing, 'Problematic' when there were some suture placement issues but it still felt secure, and 'Difficult' when there was a struggle to complete it and it looked a little ragged at the end" (30). AL rates according to these categories were $3 \%, 6 \%$ and $16 \%$, respectively (30). Given the negative impact of postoperative complications on shortterm outcomes and long-term oncological outcomes it seems clear how those results need to be prospectively investigated.

Other studies did not particularly focus on the relationship we investigated here but we include them in our discussion because they reported interesting accomplishments, which indirectly support our hypothesis, or because they appraised aspects about AL useful for the purpose of our research.

First of all, we considered an article by Rasmussen et al. which evaluated the influence of postoperative complications on the survival. A total of 133 patients were enrolled in this study cohort, with a mean age of 65 years. AL was proven by water-soluble contrast swallow, CT scan (31). Patients without complications had long-term survival of $52 \%$. On the other hand, among patients with one or more complications, long-term survival was reduced to $30 \%$ (logrank $p=0.039$ ). The most relevant factors were AL and sepsis/septic shock. However, Rasmussen et al. did not report the number of patients affected by recurrence nor the number of patients who experienced AL, considering only the impact of AL on OS; therefore, including their results in Table III was not possible. Adjusted Cox regression analysis revealed that postoperative complications were associated with an increased mortality risk with an adjusted HR of 2.02 (95\%CI $=1.1-3.7, p=0.025)$. In particular, they demonstrated how patients with AL had a significantly higher risk (adjusted for age, gender, tumor stage of a poor outcome), which was demonstrated by a higher HR of 2.37, 95\% CI=1.17-4.81, $p=0.016$.

Rizk et al. highlighted how surgical technical complications had a significant negative impact on overall survival (multivariable $\mathrm{HR}=1.41, \quad 95 \% \mathrm{CI}=1.22-1.63$, $p=0.008)$. However, Rizk et al. did not focus their research 
on $\mathrm{AL}$ in particular. On the other hand, in this study, medical complications alone had no impact on overall survival, including recurrence (15).

Conversely, Ancona et al. found no negative impact on outcomes, neither regarding medical complications alone, nor surgical complication alone, showing negative impact on oncological outcome only if such complications occurred concurrently (32).

Another article that attempted to demonstrate the possible relationship between AL and occurrence of recurrence was written by Lindner et al. (33): they wanted to assess the impact of the postoperative complications on long-term outcome in patients with esophageal cancer. The study included 134 patients who underwent transthoracic esophagectomy between 2005 and 2010. The median overall survival was sub-analyzed according to histological subtype. For those with esophageal adenocarcinoma, if anastomotic insufficiency occurred the median overall survival was 33.7 (range=3.3-91.9) months, and if not, 42.8 (range=2.6-95.3) months. For those with esophageal squamocellular carcinoma, the median overall survival was 40.04 (range=3.4-51.8) months in those who experienced AL, and 30.0 (1.4-5.2) months in those who did not (32). They did not take into consideration the recurrence rates in patients with AL. According to their results, neither anastomotic insufficiency, nor neoadjuvant treatment influenced the incidence of complications or long-term survival. The overall survival of the total population was 38.1 (range=1.4-95.3) months (33).

Another article which aimed to investigate the relationship between postoperative complications and long-term survival in patients with surgically resected esophageal squamous cell carcinoma was taken into account in our review (34). It was a single-institution study, including 502 consecutive patients. Postoperative complications of Clavien-Dindo classification grade greater than 2, occurred in 217 patients (43\%). Among those, 68 patients $(13.5 \%)$ experienced AL, but the results of their long-term clinical outcomes were combined with other complication, such as surgical site infection, recurrent nerve paralysis, cardiovascular complication. Overall, postoperative complications did not affect long-term clinical outcomes of these patients (log-rank $p=0.28$ ) (34).

Another interesting article that we selected analyzed surgical complications and long-term survival after esophagectomy in a nationwide Swedish cohort study (35). The authors wanted to evaluate how acute surgical complications after esophageal resection for cancer may reduce long-term survival or not. Eligible patients comprised those afflicted by esophageal or cardia cancer and who underwent surgical resection in Sweden in 2001-2005, including 615 patients in total. Among 567 included patients who survived at least 90 days postoperatively, 130 (22.9\%) experienced a predefined surgical complication within 30 days of surgery. They showed acute complication might be an independent predictor for poorer long-term survival. Extrapolating the data of interest, they found 47 patients afflicted by AL (7.6\%), but they did not relate this to data for recurrence. However, in the group affected by surgical complication, the adjusted HR of poor prognosis was higher compared to patients without such complications $(\mathrm{HR}=1.29$, 95\% CI $=1.021 .63$ ).

Furthermore, we also took into account a study published by Carrot et al. (36), which used the Accordion Severity Grading System (36) to categorize complications according to the treatment responses and resource use. Patients undergoing esophagectomy from 2000 to 2008 were considered, a total of 285 patients. In 156 patients $(54.7 \%)$ neoadjuvant therapy was used, and the operative mortality rate was $0.7 \%$; 144 patients (50.5\%) experienced complications. This severity grading system allowed documentation of complications and even their relevant economic impact. Cox regression multivariate analysis demonstrated an inversely proportional relationship between overall survival and occurrence of postoperative complications. Among those 285 patients, 11 were affected by AL (3.9\%). Using multivariate regression, AL was associated with increased cost (multivariate $\mathrm{B}=0.149, p=0.05$ ). However, this study did not demonstrate that individual Accordion grades affect survival or recurrence. The relatively small number of patients with higher-level Accordion grades limited the opportunity for a complete analysis (36).

Lagarde et al. looked notably only at death due to cancer recurrence, thus not using recurrence itself, concluding that postoperative medical and surgical complications were associated in an independent pattern with the timing of death due to recurrence, but not to death from recurrence itself (11). Although this occurrence of complications had affinity with our review, given the differences reported, we preferred not to include the results of their work in our table.

The last study that we have taken into consideration was carried out by Attili et al. (38). This work considered the significance of postoperative events in gastrointestinal malignancies. This was a retrospective study conducted at Kidway memorial institute of Oncology (2004-2006) which evaluated patients who had undergone surgery with curative intent for gastrointestinal malignancies. They assumed AL as risk factor predictive of poor disease-free survival and 236 were evaluated. They found that the risk of relapse was 9.8 higher in patients with AL and the risk was uniform across all types of gastrointestinal malignancies. They reported a relapse rate of $19 \%$ in AL after esophagectomy. Among risk factors for recurrence, $\mathrm{AL}$ had on $\mathrm{OR}$ of $9.8(95 \% \mathrm{CI}=4.2-$ $19.8, p=0.01)$. This study, as all the systematic reviews on such a specific and poorly studied topic, suffers from some bias; despite the wealth of studies reported in Asiatic Literature, we were unable to find an article clearly focused on the parameters we have taken into consideration for this review; one of studies included in our research (25) also 
included curative gastric resection, not only esophageal or junctional carcinoma. Hii et al. reported a total of 10 patients with R1 resection (7.2\%), which could be a potential source of bias (22). Furthermore, all the included studies were retrospective in nature, carrying an intrinsic risk of selection bias, with a small cohort of patients, and were performed over a long period of time (1998-2018), possibly including heterogeneity linked to the changes in clinical practice, technology, surgical and chemoradiotherapy protocols. Among included retrospective studies, only the work carried out by Tam et al. (26) considered propensity matching in order to reduce bias.

Indeed, changes in adjuvant chemotherapy practice may have included some selection bias, with patients treated in the most recent years showing better oncological outcomes due to more effective protocols. Moreover, the different AL evaluation among included studies might be a potential source of bias.

\section{Conclusion}

According to the studies we reported in Table III, there is a tendency to attribute recurrence to AL (23-25); in fact, three out of seven studies confirmed this relationship, and in terms of numbers, those studies are the most relevant we found in literature to date. In the study cited which demonstrated a link between leakage and recurrence, there is conformity of opinions in considering proinflammatory environment and adjuvant therapy delay as main factors involved in an increased risk of occurrence of recurrence. Given the negative impact of AL on short-term outcomes and long-term oncological outcomes, it seems clear that there needs to be further prospective investigation. In our opinion, therefore, we consider that further studies are necessary, even by putting together different case series, and also investigating biomolecular markers involved, in order to be finally able to highlight the importance of AL on the recurrence of this disease. However, although the evidence is not yet confirmed by prospective study or meta-analysis, from our review, it would seem prudent to consider closer follow-up or even more aggressive oncological therapy of patients affected by AL.

\section{Compliance with Ethical Standards}

All procedures performed in studies involving human participants were in accordance with the ethical standards of the institutional and/or national research committee and with the 1964 Helsinki declaration and its later amendments or comparable ethical standards.

\section{Conflicts of Interest}

The Authors have no conflict of interests in regard to this study.

\section{Authors' Contributions}

Giammauro Berardi, Giovanni Moschetta, Matteo Cinquepalmi, Laura Antolino, Giuseppe Nigri, Francesco D'Angelo, Stefano Valabrega, Giovanni Ramacciato contributed equally to this work, generated the figures and wrote the article. Paolo Aurello designed the aim of the review, wrote and supervised all steps in producing the article.

\section{References}

1 Smyth EC, J Lagergren J, Lordick F, Shah MA, Lagergren P and Cunningham D: Oesophageal cancer. Lancet 390: 2383-2396, 2017. PMID: 28648400. DOI: $10.1038 / n r d p .2017 .48$

2 National Comprehensive Cancer Network: Esophageal and Esophagogastric Junction Cancers. (version 1, 2017). Available at: https://www.nccn.org/professionals/physician_gls/esophagela.pdf

3 Rice TW, Patil DT and Blackstone EH: 8th edition AJCC/UICC staging of cancers of the esophagus and esophagogastric junction: Application to clinical practice. Ann Cardiothorac Surg 6(2): 119-130, 2017. DOI: 10.21037/acs.2017.03.14

4 Hulscher JB, Tijssen J Obertop $\mathrm{H}$ and van Lanschot JJ: Transthoracic versus transhiatal resection for carcinoma of the esophagus: A meta analysis. Ann Thorac Surg 72: 306-313, 2001. PMID: 11465217.

5 Wang S, Liu J, Wang S, Zhao H, Ge S and Wang W: Adverse Effects of anastomotic leakage on local recurrence and survival after curative anterior resection for rectal cancer: A systematic review and meta-analysis. World J Surg 41: 277-284, 2017. PMID: 27743072. DOI: 10.1007/s00268-016-3761-1

6 Shimada H, Fukagawa T, Haga $\mathrm{Y}$ and Oba K: Does postoperative morbidity worsen the oncological outcome after radical surgery for gastrointestinal cancers? A systematic review of the literature. Ann Gastroenterol Surg 1: 11-23, 2017. PMID: 29863169. DOI: $10.1002 /$ ags3.12002

7 Liberati A, Altman DG, Tetzlaff J, Mulrow C, Gøtzsche PC, Ioannidis JP, Clarke M, Devereaux PJ, Kleijnen J and Moher D: The PRISMA Statement for reporting systematic reviews and meta-analyses of studies that evaluated health care interventions: Explanation and elaboration. PLoS Med 6(7): e1000100, 2009. DOI: 10.1371/journal.pmed.1000100

8 Chien PF, Khan KS and Sissakos D: Registration of systematic reviews: PROSPERO. BJOG 119(8): 903-905, 2012. PMID: 22703418. DOI: $10.1111 / \mathrm{j} .1471-0528.2011 .03242 . x$

9 Wells G, Shea B, O'Connell D, Peterson J, Welch V, Losos M and Tugwell P: The Newcastle-Ottawa Scale (NOS) for assessing the quality of nonrandomised studies in meta-analyses, 2013. Available at: http://www.ohri.ca/programs/clinical_ epidemiology/nosgen.pdf

10 Higgins JPT and Green S: Cochrane Handbook for Systematic Reviews of Interventions Version 5.1.0 updated, 2011. Available at: http://handbook-5-1.cochrane.org/

11 Lagarde SM, de Boer JD, ten Kate FJ, Busch OR, Obertop H and van Lanschot JJ: Postoperative complications after esophagectomy for adenocarcinoma of the esophagus are related to timing of death due to recurrence. Ann Surg 247(1): 71-76, 2008. PMID: 18156925. DOI: 10.1097/SLA.0b013e31815b695e

12 Minamide J, Aoyama, Koizumi H, Yoneyama K, Hoshino S, Kamiya J, Tamai S and Kameda Y: Postoperative complications in patients of esophageal cancer after neoadjuvant chemotherapy. 
Jpn J Thorac Cardiovasc Surg 47(11): 542-545, 1999. PMID: 10614093. DOI: 10.1007/BF03218059

13 Luc G, Durand M, Chiche L and Collet D: Major post-operative complications predict long-term survival after esophagectomy in patients with adenocarcinoma of the esophagus. World J Surg 39(1): 216-222, 2015. PMID: 25189448. DOI: 10.1007/s00268014-2754-1

14 Hirai T, Yamashita Y, Mukaida H, Kuwahara M, Inoue H and Toge T: Poor prognosis in esophageal cancer patients with postoperative complications. Surg Today 28(6): 576-579, 1998 PMID: 9681604. DOI: 10.1007/s005950050187

15 Rizk NP, Bach PB, Schrag D, Bains MS, AD, Karpeh M, Brennan MF and Rusch VW: The Impact of complications on outcomes after resection for esophageal and gastroesophageal junction carcinoma. J Am Coll Surg 198(1): 42-50, 2004. PMID: 14698310. DOI: 10.1016/j.jamcollsurg.2003.08.007

16 Escofet X, Manjunath A Twine C, Havard TJ, Clark GW and Lewis WG: Prevalence and outcome of esophagogastric anastomotic leak after esophagectomy in a UK regional cancer network. Dis Esophagus 23: 112-116, 2010. PMID: 19549208. DOI: $10.1111 / \mathrm{j} .1442-2050.2009 .00995$

17 Xia BT, Rosato EL, Chojnacki KA, Crawford AG, Weksler B and Berger AC: Major perioperative morbidity does not affect long-term survival in patients undergoing esophagectomy for cancer of the esophagus or gastroesophageal junction. World $\mathrm{J}$ Surg 37(2): 408-415, 2013. PMID: 23052816. DOI: $10.1007 /$ s00268-012-1823-6

18 Aminian A, Panahi N. Mirsharifi R, Karimian F, Meysamie A, Khorgami Z and Alibakhshi A: Predictors and outcome of cervical anastomotic leakage after esophageal cancer surgery. Cancer Res Ther 7(4): 448-453, 2011. PMID: 22269408. DOI: 10.4103/0973-1482.92016

19 Booka E, Takeuchi H, Nishi T, Matsuda S, Kaburagi T, Fukuda K, Nakamura R, Takahashi T, Wada N, Kawakubo H, Omori T and Kitagawa Y: The Impact of postoperative complications on survivals after esophagectomy for esophageal cancer. Medicine 94(33): e1369, 2015. PMID: 26287423. DOI: 10.1097/MD 0000000000001369

20 Rutegård M, Lagergren P, Rouvelas I and Lagergren J: Intrathoracic anastomotic leakage and mortality after esophageal cancer resection: A population-based study. Ann Surg Oncol 19(1): 99-103, 2012. PMID: 21769467. DOI: 10.1245/s10434011-1926-6

21 Lerut T, Moons J, Coosemans W, Van Raemdonck D, De Leyn P, Decaluwé H, Decker G and Nafteux P: Postoperative complications after transthoracic esophagectomy for cancer of the esophagus and gastroesophageal junction are correlated with early cancer recurrence: role of systematic grading of complications using the modified Clavien classification. Ann Surg 250(5): 798-807, 2009. PMID: 19809297. DOI: 10.1097/ SLA.0b013e3181bdd5a8

22 Hii MW, Smithers BM, Gotley DC, Thomas JM, Thomson I, Martin I and Barbour AP: Impact of postoperative morbidity on long-term survival after oesophagectomy. Br J Surg 100: 95-104, 2013. PMID: 23148025. DOI: 10.1002/bjs.8973

23 Markar S, Gronnier C. Duhamel A, Mabrut JY, Bail JP, Carrere N, Lefevre JH, Brigand C, Vaillant JC, Adham M, Msika S, Demartines N, Nakadi IE, Meunier B, Collet D and Mariette C: The impact of severe anastomotic leak on long-term survival and cancer recurrence after surgical resection for esophageal malignancy. Ann Surg 262(6): 972-980, 2015. PMID: 26469952. DOI: $10.1097 /$ SLA.0000000000001011

24 Kofoed S, Calatayud D, Jensen LS, Helgstrand F, Achiam MP, De Heer P and Svendsen LB: Danish Esophageal, Cardia and Stomach Cancer Group. Intrathoracic anastomotic leakage after gastroesophageal cancer resection is associated with increased risk of recurrence. Thorac Cardiovasc Surg 150(1): 42-48, 2015. PMID: 25986493. DOI: 10.1016/j.jtcvs.2015.04.030

25 Andreou A, Biebl M, Dadras M, Struecker B, Sauer IM, ThussPatience PC, Chopra S, Fikatas P, Bahra M, Seehofer D, Pratschke J and Schmidt SC: Anastomotic leak predicts diminished long-term survival after resection for gastric and esophageal cancer. Surgery 160(1): 191-203, 2016. PMID: 27067160. DOI: $10.1016 /$ j.surg.2016.02.020

26 Tam V, Luketich JD, Winger DG, Sarkaria IS, Levy RM, Christie NA, Awais O Shende MR and Nason KS: Cancer recurrence after esophagectomy: impact of postoperative infection in propensity-matched cohorts. Ann Thorac Surg 102(5): 1638-1646, 2016. PMID: 27353482. DOI: 10.1016/ j.athoracsur.2016.04.097

27 Belmouhand M, Svendsen LB, Kofoed SC, Normann G, Baeksgaard L and Achiam MP: Recurrence following curative intended surgery for an adenocarcinoma in the gastroesophageal junction: a retrospective study. Dis Esophagus 31(4), 2018. PMID: 29228216. DOI: 10.1093/dote/dox136

28 Beecher SM, O'Leary DP, McLaughlin R, Sweeney KJ and Kerin MJ: Influence of complications following immediate breast reconstruction on breast cancer recurrence rates. Br J Surg 103(4): 391-398, 2016. PMID: 26891211. DOI: 10.1002/ bjs.10068

29 Dindo D, Demartines N and Clavien PA: Classification of surgical complications: a new proposal with evaluation in a cohort of 6336 patients and results of a survey. Ann Surg 240(2): 205-213, 2004. PMID: 15273542.

30 Wong J: Esophageal resection for cancer in The University of Hong Kong, Queen Mary Hospital, Hong Kong, China (19822008). J Thorac Dis 10(Suppl 16): S1843-S1844, 2018. PMID: 30026969. DOI: $10.21037 /$ jtd 2018.01 .84

31 Rasmussen SR, Nielsen VR, Fenger AS, Siemsen M and Ravn HB: Postoperative complications and survival after surgical resection of esophageal squamous cell carcinoma. J Thorac Dis 10(7): 4052-4060, 2018. PMID: 30174848. DOI: $10.21037 /$ jtd .2018 .07 .04

32 Ancona E, Cagol M, Epifani M, Cavallin F, Zaninotto G, Castoro C, Alfieri R and Ruol A: Surgical complications do not affect long-term survival after esophagectomy for carcinoma of the thoracic esophagus and cardia. J Am Coll Surg 203(5): 661-669, 2006. PMID: 17084327. DOI: 10.1016/j.jamcollsurg.2006.07.017

33 Lindner K, Fritz M, Haane C, Senninger N, Palmes D and Hummel R: Postoperative complications do not affect long-term outcome in esophageal cancer patients. World J Surg 38(10): 2652-2661, 2014. PMID: 24867467. DOI: 10.1007/s00268-0142590-3

34 Baba Y, Yoshida N, Shigaki H, Iwatsuki M, Miyamoto Y, Sakamoto Y, Watanabe M and Baba H: Prognostic impact of postoperative complications in 502 patients with surgically resected esophageal squamous cell carcinoma: A retrospective single-institution study. Ann Surg 264(2): 305-311, 2016. PMID: 26670288. DOI: $10.1097 /$ SLA.0000000000001510

35 Rutegård M, Lagergren P, Rouvelas I, Mason R and Lagergren J: Surgical complications and long-term survival after 
esophagectomy for cancer in a nationwide Swedish cohort study. Eur J Surg Oncol 38(7): 555-561, 2012. PMID: 22483704. DOI: 10.1016/j.ejso.2012.02.177

36 Carrott PW, Markar SR Kuppusamy MK, Traverso LW and Low DE: Accordion severity grading system: assessment of relationship between costs, length of hospital stay, and survival in patients with complications after esophagectomy for cancer. J Am Coll Surg 215(3): 331-336, 2012. PMID: 22683069. DOI: 10.1016/j.jamcollsurg.2012.04.030

37 Strasberg SM, Linehan DC and Hawkins WG: The accordion severity grading system of surgical complications. Ann Surg 250(2): 177-186, 2009. PMID: 19638919. DOI: 10.1097/ SLA.0b013e3181afde41
38 Attili VS, Bapsy PP, Ramachandra C, Reddy CO, Prabhakaran PS, Varma PP and Chandra VR: Influence of postoperative complications on relapse-free survival in gastrointestinal malignancies. Gastrointest Cancer Res 3(5): 179-182, 2009. PMID: 20084159.
Received February 26, 2019

Revised March 18, 2019

Accepted March 20, 2019 